\title{
Hammerstein equations with an integral over a noncompact domain
}

\author{
by Robert Stańczy (Łódź)
}

\begin{abstract}
The existence of solutions of Hammerstein equations in the space of bounded and continuous functions is proved. It is obtained by the Schauder fixed point theorem using a compactness theorem. The result is applied to Wiener-Hopf equations and to ODE's.
\end{abstract}

1. Introduction. In 1929 Hammerstein published paper [4] concerning nonlinear integral equations with integral operators of the form

$$
S x(t)=\int_{T} G(t, s) r(x(s), s) d s .
$$

If the domain of integration is compact and the functions $G$ and $r$ are continuous, then equation (1) can be considered in the space $C(T)$ of continuous functions. It can also be considered in the space $L^{p}(T)$ of integrable functions (where $T$ has a finite or an infinite measure).

In this paper, a noncompact domain of integration is considered. We work in the space of bounded and continuous functions. Therefore, to apply the Schauder theorem, we need sufficient conditions for compactness in this space. Some of them are presented in [1]. In our paper, however, necessary and sufficient conditions are stated. The main theorem gives conditions for the complete continuity of the Hammerstein operator. It is the extension of the results of [5] where $T=(-\infty, \infty)$. Some applications to the WienerHopf equation on a half-line or on a half-space are given. The theorem can also be applied to ODE's of second order with boundedness as a boundary condition since the Green function is continuous.

2. Preliminaries and auxiliary lemmas. Let $X$ be a Banach space with norm $\|\cdot\|$ and let $T$ be a locally compact metric space countable at

1991 Mathematics Subject Classification: 45G10, 47H30.

Key words and phrases: Hammerstein operator, Wiener-Hopf equation. 
infinity, i.e. $T=\bigcup_{i=1}^{\infty} K_{i}$ where $\left(K_{i}\right)_{i \in \mathbb{N}}$ are compact and satisfy $K_{i} \subset$ $\operatorname{int}\left(K_{i+1}\right)$ for any $i \in \mathbb{N}$.

For $V \subset X$ let $\overline{\operatorname{conv}} V$ denote the smallest convex, closed set of $X$ containing $V$. We write $K \Subset T$ if $K \subset T$ is compact.

We shall also use the following function spaces:

- $L(X)$ - the space of bounded linear operators from $X$ to $X$,

- $B C(T, X)$ - the space of bounded continuous functions from $T$ to $X$ with sup norm $\|\cdot\|_{\infty}$,

- $C(K, X)$ (where $K \Subset T)$ - the space of continuous functions with sup norm denoted as above,

- $C C(X, X)$-the space of completely continuous functions from $X$ to $X$, i.e. continuous and compact (mapping bounded subsets of $X$ into relatively compact ones), with metric

$$
d(f, g)=\sum_{n=1}^{\infty} 2^{-n} \frac{\sup _{\|x\| \leq n}\|f(x)-g(x)\|}{1+\sup _{\|x\| \leq n}\|f(x)-g(x)\|} .
$$

The space $C C(X, X)$ thus defined is a Fréchet space, i.e. locally convex and completely metrizable. The convergence in this space is the uniform convergence on bounded subsets of $X$.

For $y \in X$ and $x \in B C(T, X)$, we write $\lim _{t \rightarrow \infty} x(t)=y$ if

$$
\forall_{\varepsilon>0} \exists_{K \Subset T} \forall_{t \in T \backslash K} \quad\|x(t)-y\|<\varepsilon
$$

or one can understand it in terms of the Alexander compactification of the space $T$ with the point $\infty$.

We shall also use the Bochner integral over the domain $T$. Let $\mu$ denote a measure on $\mathcal{B}(T)$ (the $\sigma$-algebra of Borel subsets of $T$ ), finite on compact subsets of $T$. For the Bochner integral of vector-valued functions see [3, pp. 44-52] or [6, pp. 132-136].

Let us recall a property of the Bochner integral to be used in the sequel.

Theorem 1. Let $x: T \rightarrow X$ be a Bochner integrable function. Then, for each set $K \subset T$ of finite measure, we have

$$
\int_{K} x(t) d t \in \mu(K) \overline{\operatorname{conv}}(x(K)) .
$$

Pr o of. See [3, Corollary 8, p. 48].

Now, we prove some auxiliary lemmas concerning the Nemytskiur operator. Let $r: X \times T \rightarrow X$.

Lemma 1. Suppose that the mapping $T \ni t \mapsto r(\cdot, t) \in C C(X, X)$ is well defined and continuous. Then $r: X \times T \rightarrow X$ is continuous.

Proof. Fix $t_{1} \in T, x_{1} \in X$ and $\varepsilon>0$. There exist $\delta, \gamma>0$ such that $d\left(t, t_{1}\right)<\delta$ implies $d\left(r(\cdot, t), r\left(\cdot, t_{1}\right)\right)<\varepsilon / 2$, whence $\left\|r\left(x, t_{1}\right)-r\left(x_{1}, t_{1}\right)\right\|$ 
$<\varepsilon / 2$ for any $\left\|x-x_{1}\right\|<\gamma$. Then, for $\left\|x-x_{1}\right\|<\gamma$ and $d\left(t, t_{1}\right)<\delta$, we get

$$
\begin{aligned}
\left\|r(x, t)-r\left(x_{1}, t_{1}\right)\right\| & \leq\left\|r(x, t)-r\left(x, t_{1}\right)\right\|+\left\|r\left(x, t_{1}\right)-r\left(x_{1}, t_{1}\right)\right\| \\
& \leq \varepsilon / 2+\varepsilon / 2=\varepsilon .
\end{aligned}
$$

Lemma 2. Suppose $r: X \times T \rightarrow X$ is continuous. Let $K \Subset T, x \in$ $C(K, X)$ and $\varepsilon>0$. Then there exists $\delta>0$ such that, for any $t \in K$ and $y \in C(K, E)$ satisfying $\|y(t)-x(t)\|<\delta$, we have $\|r(y(t), t)-r(x(t), t)\|<\varepsilon$.

P r o of. Suppose that, on the contrary, there exist $x_{0} \in C(K, X), \varepsilon_{0}>0$, $t_{n} \in K$ and $y_{n} \in C(K, X)$ such that $\left\|y_{n}\left(t_{n}\right)-x_{0}\left(t_{n}\right)\right\|<1 / n$ and

$$
\left\|r\left(y_{n}\left(t_{n}\right), t_{n}\right)-r\left(x\left(t_{n}\right), t_{n}\right)\right\| \geq \varepsilon_{0} .
$$

From the sequence $t_{n} \in K$ we can extract a convergent subsequence $t_{n_{k}} \rightarrow t_{0}$, whence $x_{0}\left(t_{n_{k}}\right) \rightarrow x_{0}\left(t_{0}\right)$ and $\left\|y_{n_{k}}\left(t_{n_{k}}\right)-x\left(t_{n_{k}}\right)\right\|<1 / n_{k}$. Hence $y_{n_{k}}\left(t_{n_{k}}\right) \rightarrow x_{0}\left(t_{0}\right)$. Thus $r\left(y_{n_{k}}\left(t_{n_{k}}\right), t_{n_{k}}\right)-r\left(x_{0}\left(t_{n_{k}}\right), t_{n_{k}}\right) \rightarrow 0$, which contradicts our assumption.

LEMma 3. Let the mapping $T \ni t \mapsto r(\cdot, t) \in C C(X, X)$ be continuous. Then, for any $M>0$ and any $K \Subset T$, the set $\{r(x, t):\|x\| \leq M, t \in K\}$ is relatively compact.

Proof. Fix $M>0$. By the complete continuity of $r(\cdot, t)$ for any $t \in K$, we can choose an $\varepsilon / 2$-net $\left(r\left(x_{i}^{t}, t\right)\right)_{i=1}^{k_{t}}$ of the set $\{r(x, t):\|x\| \leq M\}$. Define $\widetilde{r}: T \rightarrow C C(X, X)$ by $\widetilde{r}(t)=r(\cdot, t)$. Since $\widetilde{r} \mid K$ is uniformly continuous, we may choose $\delta>0$ such that $d\left(s, s^{\prime}\right)<\delta$ implies $d\left(\widetilde{r}(s), \widetilde{r}\left(s^{\prime}\right)\right)<\varepsilon / 2$. Let $s_{1}, \ldots, s_{p}$ be a $\delta$-net of $K$. Then $\left(r\left(x_{i}^{s_{j}}, s_{j}\right)\right)_{i=1 j=1}^{k_{s_{j}} p}$ is an $\varepsilon$-net of the set $\{r(x, t):\|x\| \leq M, t \in K\}$. Indeed, for any $s \in K$ and $\|x\| \leq M$, we may choose $s_{j}$ such that $d\left(s, s_{j}\right)<\delta$ and $x_{i}^{s_{j}}$ such that

$$
\left\|r\left(x_{i}^{s_{j}}, s_{j}\right)-r\left(x, s_{j}\right)\right\| \leq \varepsilon / 2 .
$$

Then

$$
\begin{aligned}
\left\|r\left(x_{i}^{s_{j}}, s_{j}\right)-r(x, s)\right\| & \leq\left\|r\left(x_{i}^{s_{j}}, s_{j}\right)-r\left(x, s_{j}\right)\right\|+\left\|r\left(x, s_{j}\right)-r(x, s)\right\| \\
& \leq \varepsilon / 2+\varepsilon / 2=\varepsilon .
\end{aligned}
$$

Lemma 4. Let $M>0$. Suppose that the mapping $T \ni t \mapsto r(\cdot, t) \in$ $C C(X, X)$ is continuous, and that there exists $b \in B C(T, X)$ such that

$$
\lim _{t \rightarrow \infty} \sup _{\|x\| \leq M}\|r(x, t)-b(t)\|=0 .
$$

Then $r(B(0, M) \times T)$ is bounded in $X(B(0, M)$ is the closed ball with centre 0 and radius $M>0)$.

Proof. Fix $\varepsilon>0$ and choose $K \Subset T$ such that $\|x\| \leq M, t \notin K$ imply

$$
\|r(x, t)-b(t)\| \leq \varepsilon\|r(x, t)\| \leq \varepsilon+\|b(t)\| .
$$


By Lemma 3, $T_{K, M}=\sup _{\|x\| \leq M, t \in K}\|r(x, t)\|<\infty$, whence

$$
\|r(x, t)\| \leq \max \left\{\varepsilon+\|b\|_{\infty}, T_{K, M}\right\} .
$$

\section{Main results}

Theorem 2. Let $T$ be a metric, locally compact space countable at $\infty$ and let $X$ be a Banach space. Then the relative compactness of the set $F \subset B C(T, X)$ is equivalent to the conjunction of three conditions:

$1^{\mathrm{o}}$ The set $\{x(t): x \in F\}$ is relatively compact in $X$ for each $t \in T$.

$2^{\circ}$ For each $K \Subset T$ the functions in $F_{K}=\{x \mid K: x \in F\}$ are equicontinuous.

$3^{\circ}$ For each $\varepsilon>0$, there exist $\delta>0$ and $K \Subset T$ such that, for any $x, y \in F$, if $\|x|K-y| K\|_{\infty} \leq \delta$, then $\|x-y\|_{\infty} \leq \varepsilon$.

Proof. Necessity. If $F \subset B C(T, X)$ is relatively compact then the Ascoli-Arzelà theorem implies $1^{\circ}$ and $2^{\circ}$. If $3^{\circ}$ were not satisfied, there would be $\varepsilon_{0}>0$ and sequences $\left(x_{n}\right),\left(y_{n}\right),\left(K_{n}\right)$ such that

$$
\left\|x_{n}\left|K_{n}-y_{n}\right| K_{n}\right\|_{\infty} \leq \frac{1}{n} \quad \text { and }\left\|x_{n}-y_{n}\right\|_{\infty}>\varepsilon_{0} .
$$

We may extract a convergent subsequence $x_{n_{k}}-y_{n_{k}} \rightarrow x-y$. But then $x\left|K_{n}=y\right| K_{n}$ for each $n \in \mathbb{N}$, which contradicts $\|x-y\|_{\infty} \geq \varepsilon_{0}$.

Sufficiency. Take $\varepsilon>0$ and choose $K \Subset T$ from condition $3^{\circ}$. By the Ascoli-Arzelà theorem the set $\{x \mid K\}$ has a finite $\delta$-net: $\left\{x_{1}\left|K, \ldots, x_{l}\right| K\right\}$. Then, from $3^{\circ}$ we see that $\left\{x_{1}, \ldots, x_{l}\right\}$ is an $\varepsilon$-net for $F$.

REMARK 1 . The above theorem, with a similar proof, can be extended to the case of $X$ being a complete, metric space.

REMARK 2. For $T=[0, \infty)$, some sufficient conditions for compactness in $B C([0, \infty)$ are stated in [1] with the use of measures of noncompactness.

Remark 3. Condition $3^{\circ}$ is satisfied if, for any $x, y \in F$,

$$
\lim _{t \rightarrow \infty}\|x(t)-y(t)\|=0 .
$$

Example. Let $T=X=\mathbb{R}, F=\{\sin (x), \cos (x)\}$. Then $F$ is compact in $B C(T, X)$ though the condition of Remark 3 is not satisfied.

THEOREM 3. Let $T$ be a metric, locally compact space countable at $\infty$ and let $X$ be a Banach space. Suppose $T$ is equipped with a measure on the $\sigma$-algebra of Borel subsets of $T$, finite on compact sets. Define an integral operator $S$ on $B C(T, X)$ by

$$
S x(t)=\int_{T} G(t, s) r(x(s), s) d s .
$$


Assume that

(i) $G: T \times T \rightarrow L(X)$ is continuous,

(ii) $\sup _{t \in T} \int_{T}\|G(t, s)\| d s<\infty$,

(iii) $\forall_{K \Subset T} \lim _{t \rightarrow \infty} \int_{K}\|G(t, s)\| d s=0$,

(iv) $\forall_{\varepsilon>0} \forall_{\widehat{K} \Subset T} \exists_{K \Subset T} \forall_{t \in \widehat{K}} \int_{T \backslash K}\|G(t, s)\| d s<\varepsilon$,

(v) the map $t \mapsto r(\cdot, t) \in C C(X, X)$ is continuous,

(vi) there exists a function $b \in B C(T, X)$ such that, for each $M>0$,

$$
\lim _{t \rightarrow \infty} \sup _{\|x\| \leq M}\|r(x, t)-b(t)\|=0 .
$$

Then $S$ maps $B C(T, X)$ into $B C(T, X)$ and is completely continuous.

Proof. Define $L=\sup _{t \in T} \int_{T}\|G(t, s)\| d s$. By (ii), $L<\infty$. The assumptions on $r$ imply its boundedness. Hence we get the existence of the integral $\int_{T} G(t, s) r(x(s), s) d s$ and the boundedness of the operator $S$ on any bounded set.

To get the continuity of $S x$, we divide $T$ into a compact set $K$ and apply the continuity of the function $G$ there, and the noncompact set $T \backslash K$ where we use (iv). Similarly, using (vi) on the noncompact set and (v) on the compact one, we estimate the norm $\|S x(t)-S y(t)\|$. Taking into account (ii) we get the continuity of the operator $S$.

Now, we show that $S$ is compact, i.e. for each $M>0$, the set $S(B(0, M))$ is relatively compact. In order to use the compactness criterion in $B C(T, X)$, we have to show that conditions $1^{\circ}-3^{\circ}$ are satisfied.

$1^{\circ}$ Fix $t_{0} \in T$ and $\varepsilon>0$. We shall find a finite $\varepsilon$-net for $A:=\left\{S x\left(t_{0}\right)\right.$ : $\|x\|<M\}$. Choose $K \Subset T$ such that $\|r(x, t)-b(t)\| \leq \varepsilon /(2 L)$ for $\|x\|<M$ and $t \in T \backslash K$. Set $x_{0}=\int_{T \backslash K} G\left(t_{0}, s\right) b(s) d s$. Consider

$$
B:=\left\{\int_{K} G\left(t_{0}, s\right) r(x(s), s) d s:\|x\|_{\infty} \leq M\right\} .
$$

From Lemma 3 we see that the set $Z_{t_{0}}:=\left\{G\left(t_{0}, s\right) r(x, s): s \in K,\|x\| \leq M\right\}$ is relatively compact in $X$. But the integrals in $B$ belong to the convex hull $\mu(K) \overline{\operatorname{conv}} Z_{t_{0}}$, so by the Mazur theorem, there exists a finite $\varepsilon / 2$-net $x_{1}, \ldots, x_{p}$ of $B$. We have thus obtained an $\varepsilon$-net $x_{0}+x_{1}, \ldots, x_{0}+x_{p}$ of $A$. Indeed,

$$
\begin{aligned}
\left\|S x\left(t_{0}\right)-\left(x_{0}+x_{j}\right)\right\| \leq & \left\|\int_{K} G\left(t_{0}, s\right) r(x(s), s) d s-x_{j}\right\| \\
& +\int_{T \backslash K}\left\|G\left(t_{0}, s\right)\right\| \cdot\|r(x(s), s)-b(s)\| d s \\
\leq & \frac{\varepsilon}{2}+L \frac{\varepsilon}{2 L}=\varepsilon .
\end{aligned}
$$


$2^{\circ}$ Let $\varepsilon>0$ and $\widehat{K} \Subset T$. Choose, by (iv) and (vi), $K \Subset T$ such that

whence

$$
\|r(x, s)-b(s)\| \leq\|b\|_{\infty},
$$

and

$$
\|r(x, s)\| \leq 2\|b\|_{\infty}
$$

$$
\int_{T \backslash K}\|G(t, s)\| d s \leq \varepsilon /\left(8\|b\|_{\infty}\right) \quad \text { for any } t \in \widehat{K} .
$$

Moreover, using the uniform continuity of $G$ on $\widehat{K} \times K$, we can choose some $\delta>0$ such that, for any $t, t_{1} \in \widehat{K}$ with $d\left(t, t_{1}\right)<\delta$ we have $\| G(t, s)-$ $G\left(t_{1}, s\right) \| \leq \varepsilon /(2 \mu(K) T)$. Then

$$
\begin{aligned}
\left\|S x(t)-S x\left(t_{1}\right)\right\| & \\
\leq & \int_{K}\left\|G(t, s)-G\left(t_{1}, s\right)\right\| \cdot\|r(x(s), s)\| d s \\
& \quad+\int_{T \backslash K}\left\|G(t, s)-G\left(t_{1}, s\right)\right\| \cdot\|r(x(s), s)\| d s \\
\leq & ((\mu(K) \cdot \varepsilon) /(2 \mu(K) T)) \cdot T+\left(2 \varepsilon /\left(8\|b\|_{\infty}\right)\right) \cdot 2\|b\|_{\infty}=\varepsilon .
\end{aligned}
$$

$3^{\circ}$ Let $\varepsilon>0$. From (vi), we can choose $K \Subset T$ such that, for any $s \in T \backslash K$ and $\|x\| \leq M$ we have $\|r(x, s)-b(s)\| \leq \varepsilon /(4 L)$. Moreover, from (iii) we can choose $\widehat{K} \Subset T$ such that $\int_{K}\|G(t, s)\| d s \leq \varepsilon /(4 T)$ for $t \in T \backslash \widehat{K}$. Then, for those $t$ and $\|x\|_{\infty} \leq M,\|y\|_{\infty} \leq M$, we get

$$
\begin{aligned}
\| S x(t)- & S y(t) \| \\
\leq & \int_{K}\|G(t, s)\| \cdot\|r(x(s), s)-r(y(s), s)\| d s \\
& \quad+\int_{T \backslash K}\|G(t, s)\|(\|r(x(s), s)-b(s)\|+\|r(y(s), s)-b(s)\|) d s \\
\leq & (\varepsilon /(4 T)) \cdot 2 T+2 L \cdot(\varepsilon /(4 L))=\varepsilon .
\end{aligned}
$$

THEOREM 4. Let $G$ and $r$ satisfy the assumptions of the previous theorem. Moreover, suppose that

$$
R=\limsup _{\|x\| \rightarrow \infty} \sup _{t \in T} \frac{\|r(x, t)\|}{\|x\|}<1 / L
$$

where $L=\sup _{t \in T} \int_{T}\|G(t, s)\| d s$. Then the integral equation $S x=x$ has a solution in the space $B C(T, X)$.

Proof. By assumption (2), for $\varepsilon=1 / L-R>0$, choose $M>0$ such that $\|r(x, t)\| /\|x\|<R+\varepsilon=1 / L$ for any $t \in T$ and $\|x\|>M$. Define $T=\sup _{\|x\| \leq M, t \in T}\|r(x, t)\|$. Using Lemma 4 , we get $T<\infty$. Hence, for 


$$
\begin{aligned}
\|x\|_{\infty} \leq \max \{L T, M\}, & \\
\|S x\|_{\infty} \leq & \sup _{t \in T}\left(\int_{\{s:\|x(s)\| \leq M\}}\|G(t, s)\| \cdot\|r(x(s), s)\| d s\right. \\
& \left.+\int_{\{s:\|x(s)\|>M\}}\|G(t, s)\| \cdot\|r(x(s), s)\| d s\right) \\
\leq & \sup _{t \in T}\left(\int_{\{s:\|x(s)\| \leq M\}}\|G(t, s)\| \max \{T, M / L\} d s\right. \\
& \left.+\int_{\{s:\|x(s)\|>M\}}\|G(t, s)\|(\max \{L T, M\} / L) d s\right) \\
= & \sup _{t \in T} \int_{T}\|G(t, s)\| \max \{T, M / L\} d s \\
\leq & L \max \{T, M / L\}=\max \{T L, M\} .
\end{aligned}
$$

Then $S: B(0, \max \{T L, M\}) \rightarrow B(0, \max \{T L, M\})$ and, by the Schauder theorem, using Theorem 3 , we obtain a fixed point for $S$.

Corollary. Let $T$ be a closed cone in a Banach space and let $r: X \times$ $T \rightarrow X$ satisfy conditions (v), (vi) of Theorem 3 and condition (2). Let $G: T \times T \rightarrow L(X)$ be of the form $G(t, s)=H(t-s)$ for any $t, s \in T$, where $H: T-T \rightarrow L(X)$ (- stands for algebraic difference here) is a given continuous function such that the integral $\int_{T-T}\|H(t)\| d t$ is finite. Then the integral equation $S x=x$ has a solution in the space $B C(T, X)$.

4. Applications to Wiener-Hopf equations. The last corollary from the previous section can be easily applied to nonlinear Wiener-Hopf equations on a half-line $(T=[0, \infty))$ or on a closed half-space $\left(T=\mathbb{R}^{k-1} \times\right.$ $[0, \infty))$, which will be illustrated by the following theorems.

Theorem 5. Consider the equation

$$
\int_{0}^{\infty} H(t-s) r(x(s), s) d s=x(t)
$$

where $H: \mathbb{R} \rightarrow \mathbb{R}$ is continuous and integrable, and $r: \mathbb{R} \times[0, \infty) \rightarrow \mathbb{R}$ is continuous. Moreover, if $r$ satisfies (vi) of Theorem 3 and

$$
\limsup _{|x| \rightarrow \infty} \sup _{t \in[0, \infty)} \frac{|r(x, t)|}{|x|}<\frac{1}{L} \quad \text { where } L=\int_{-\infty}^{\infty}|H(t)| d t,
$$

then equation (3) has a bounded and continuous solution $x$.

REMARK. The conditions imposed on $r$ are, in particular, satisfied if $r(x, t)=f(x) c(t)+b(t)$ where $f: \mathbb{R} \rightarrow \mathbb{R}$ and $c, b:[0, \infty) \rightarrow \mathbb{R}$ are continuous 
and satisfy $\lim _{t \rightarrow \infty}\|c(t)\|=0$ and $\lim _{\|x\| \rightarrow \infty}\|f(x)\| /\|x\|=0$.

Theorem 6. Let $\mathbb{R}_{+}^{k}=\left\{t=\left(t_{1}, \ldots, t_{k}\right) \in \mathbb{R}^{k}: t_{k} \geq 0\right\}$. Consider the equation

$$
\int_{\mathbb{R}_{+}^{k}} H(t-s) r(x(s), s) d s=x(t)
$$

where $r: \mathbb{R} \times \mathbb{R}_{+}^{k} \rightarrow \mathbb{R}$ and $H: \mathbb{R}^{k} \rightarrow \mathbb{R}$ are continuous. Moreover, if $r$ satisfies (vi) of Theorem 3 and

$$
\limsup _{|x| \rightarrow \infty} \sup _{t \in[0, \infty)} \frac{|r(x, t)|}{|x|}<\frac{1}{L} \quad \text { where } \quad L=\int_{\mathbb{R}^{k}}|H(t)| d t<\infty,
$$

then equation (4) has a bounded and continuous solution $x$.

REMARK. The conditions imposed on $r$ are satisfied if, in particular, $r(x, t)=f(x) c(t)+b(t)$ where $f: \mathbb{R} \rightarrow \mathbb{R}$ and $b, c: \mathbb{R}_{+}^{k} \rightarrow \mathbb{R}$ are continuous and satisfy $\lim _{t \rightarrow \infty}|c(t)|=0$ and $\lim _{|x| \rightarrow \infty}|f(x)| /|x|=0$.

5. Applications to ODE's. Let $T=\mathbb{R}$ and let $X$ be a Banach space. Assume that $r: X \times \mathbb{R} \rightarrow X$ is continuous and $A \in L(X)$ is such that $\operatorname{Sp}(A) \cap\{\alpha \in \mathbb{R}: \alpha \leq 0\}=\emptyset$. Consider the following boundary value problem in $X$ :

$$
\begin{aligned}
& x^{\prime \prime}=A x+r(x, t), \\
& x \text { bounded on } \mathbb{R} .
\end{aligned}
$$

The boundedness of solutions plays here the role of a boundary condition.

Under some additional assumptions on $r$, problem (5) will be transformed to an integral equation of Hammerstein type and, for the latter, Theorem 4 will be applied to obtain the existence of solutions.

First, let us recall the notion of the Green function for problem (5). The Green function for (5) is a function $G: \mathbb{R} \times \mathbb{R} \rightarrow L(X)$ satisfying the following conditions:

(i) For any $s \in \mathbb{R}, G(\cdot, s)$ satisfies the linear equation, i.e. $\frac{\partial^{2} G}{\partial t^{2}}(t, s)=$ $A G(t, s)$ for any $t \neq s$,

(ii) $\lim _{s \rightarrow t^{-}} \frac{\partial G}{\partial t}(t, s)-\lim _{s \rightarrow t^{+}} \frac{\partial G}{\partial t}(t, s)=I$ for any $t \in \mathbb{R}$,

(iii) $G(\cdot, s)$ satisfies the boundary condition for any $s \in \mathbb{R}$ (i.e. is bounded on $\mathbb{R})$.

If we have such a function it is easy to see that any solution of the integral equation

$$
x(t)=\int_{-\infty}^{\infty} G(t, s) r(x(s), s) d s
$$


satisfies the boundary value problem (5).

Indeed, integrating and using the properties of the Green function, we obtain

$$
\begin{aligned}
x^{\prime \prime}(t)= & \frac{\partial}{\partial t}\left(\int_{-\infty}^{t} \frac{\partial G}{\partial t}(t, s) r(x(s), s) d s+\frac{\partial}{\partial t}\left(\int_{t}^{\infty} \frac{\partial G}{\partial t}(t, s) r(x(s), s) d s\right)\right) \\
= & \int_{-\infty}^{\infty} \frac{\partial^{2} G}{\partial t^{2}}(t, s) r(x(s), s) d s \\
& +\left(\lim _{s \rightarrow t^{-}} \frac{\partial G}{\partial t}(t, s)-\lim _{s \rightarrow t^{+}} \frac{\partial G}{\partial t}(t, s)\right) r(x(t), t) \\
= & A x(t)+r(x(t), t) .
\end{aligned}
$$

The Green function for (5) will now be constructed. Using the Dunford operational calculus from [6, pp. 225-228], we can define the operators

$$
\begin{aligned}
A^{-1 / 2} & =-\frac{1}{2 \pi i} \int_{\Gamma} \lambda^{-1 / 2}(A-\lambda I)^{-1} d \lambda, \\
-A^{1 / 2} & =\frac{1}{2 \pi i} \int_{\Gamma} \lambda^{1 / 2}(A-\lambda I)^{-1} d \lambda, \\
\exp \left(-A^{1 / 2}\right) & =-\frac{1}{2 \pi i} \int_{\Gamma} \exp \left(-\lambda^{1 / 2}\right)(A-\lambda I)^{-1} d \lambda,
\end{aligned}
$$

where $\Gamma$ is a Jordan curve around $\operatorname{Sp}(A)$ with counterclockwise orientation. Now, we define

$$
G(t, s)=-\frac{1}{2} A^{-1 / 2} \exp \left(-A^{1 / 2}|t-s|\right) .
$$

From [6, Corollary 3, pp. 245-246] one gets

$$
\frac{\partial^{2} G}{\partial t^{2}}(t, s)=-\frac{1}{2} A^{1 / 2} \exp \left(-A^{1 / 2}(t-s)\right)=A G(t, s) \quad \text { for } t \neq s .
$$

Since $\operatorname{Sp}\left(-A^{1 / 2}\right) \subset\{\alpha \in \mathbb{C}: \operatorname{Re} \alpha<0\}$, from [2, Theorem 4.1, pp. 42-43] we get

$$
\|G(t, s)\| \leq N e^{-v|t-s|}
$$

for some constants $N, v>0$. This implies the boundedness of the function $G(\cdot, s)$ for any $s \in T$. Finally, from [6, Theorem, p. 226] and [6, Corollary 3, pp. 245-246] we obtain

$$
\begin{aligned}
\lim _{s \rightarrow t^{-}} \frac{\partial G}{\partial t}(t, s)-\lim _{s \rightarrow t^{+}} \frac{\partial G}{\partial t}(t, s)= & \lim _{s \rightarrow t^{-}} \frac{\partial}{\partial t}\left(-\frac{1}{2} A^{-1 / 2} \exp \left(-A^{1 / 2}(t-s)\right)\right) \\
& -\lim _{s \rightarrow t^{+}} \frac{\partial}{\partial t}\left(-\frac{1}{2} A^{-1 / 2} \exp \left(-A^{1 / 2}(s-t)\right)\right)
\end{aligned}
$$




$$
\begin{aligned}
& =\lim _{s \rightarrow t^{-}} \frac{1}{2} \exp \left(-A^{1 / 2}(t-s)\right)-\lim _{s \rightarrow t^{+}}-\frac{1}{2} \exp \left(-A^{1 / 2}(s-t)\right) \\
& =\frac{1}{2} I+\frac{1}{2} I=I .
\end{aligned}
$$

We have thus shown that $G$ is the Green function for problem (5) and any solution of the integral equation (6) satisfies the boundary value problem (5). Now, we are ready to formulate the existence theorem for (5).

Theorem 7. Assume that $A \in L(X)$ is such that $\operatorname{Sp}(A) \cap\{\alpha \in \mathbb{R}$ : $\alpha \leq 0\}=\emptyset$ and $r(x, t)=c(t) f(x)+b(t)$ where $c: \mathbb{R} \rightarrow \mathbb{R}$ is continuous, $\lim _{|t| \rightarrow \infty}|c(t)|=0, b: \mathbb{R} \rightarrow X$ is continuous and bounded, and $f: X \rightarrow X$ is completely continuous. Then BVP (5) has at least one solution provided

$$
\limsup _{\|x\| \rightarrow \infty} \frac{\|f(x)\|}{\|x\|}<\frac{v}{2 N\|c\|_{\infty}} .
$$

Proof. To prove the existence of solutions for the integral equation (6), we have to show that the integral operator $S$ defined by the right-hand side of (6) satisfies the assumptions of Theorem 4.

First, we show that the assumptions of Theorem 3, implying the complete continuity of $S$, are satisfied.

From inequality (7) it follows that conditions (ii)-(iv) of Theorem 3 are satisfied. Assumption (v) is also satisfied since the functions $b, c$ are continuous and $f$ maps bounded subsets of $X$ into relatively compact ones. The last assumption (vi) is satisfied since

$$
\begin{aligned}
\lim _{t \rightarrow \infty} \sup _{\|x\| \leq M}\|r(x, t)-b(t)\| & =\lim _{t \rightarrow \infty} \sup _{\|x\| \leq M}\|c(t) f(x)\| \\
& \leq \sup _{\|x\| \leq M}\|f(x)\| \lim _{t \rightarrow \infty}|c(t)|=0 .
\end{aligned}
$$

Now, we show that condition (2) of Theorem 4 is satisfied. From inequality (7) we get

$$
L=\sup _{t \in \mathbb{R}} \int_{-\infty}^{\infty}\|G(t, s)\| d s \leq N \sup _{t \in \mathbb{R}}\left(\int_{-\infty}^{t} e^{-v(t-s)} d s+\int_{t}^{\infty} e^{-v(s-t)} d s\right)=\frac{2 N}{v} .
$$

Then

$$
\lim _{\|x\| \rightarrow \infty} \sup _{t \in T} \frac{\|c(t) f(x)+b(t)\|}{\|x\|} \leq\|c\|_{\infty} \varlimsup_{\|x\| \rightarrow \infty} \frac{\|f(x)\|}{\|x\|}<\frac{v}{2 N}=\frac{1}{L} .
$$

From Theorem 4 we get the assertion.

Analogously we obtain the existence of a solution of the boundary value problem on a half-line.

Let $T=\left[t_{0}, \infty\right)$ and let $X$ be a Banach space. Assume that $r: X \times$ $\left[t_{0}, \infty\right) \rightarrow X$ is continuous and $A \in L(X)$ satisfies $\operatorname{Sp}(A) \cap\{\alpha \in \mathbb{R}:$ 
$\alpha \leq 0\}=\emptyset$. Consider the boundary value problem

$$
\begin{aligned}
& x^{\prime \prime}=A x+r(x, t), \\
& x\left(t_{0}\right)=0, \\
& x \text { bounded on }\left[t_{0}, \infty\right) .
\end{aligned}
$$

Define

$$
G(t, s)=\frac{1}{2} A^{-1 / 2}\left(\left(\exp \left(-A^{1 / 2}\left(s+t-2 t_{0}\right)\right)-\exp \left(-A^{1 / 2}|t-s|\right)\right) .\right.
$$

From [2, Theorem 4.1, pp. 42-43] we get

$$
\|G(t, s)\| \leq N_{1} e^{-v\left(t+s-2 t_{0}\right)}+N_{2} e^{-w|t-s|}
$$

for some constants $N_{1}, N_{2}, v, w>0$. As in the previous case, one can verify that $G$ is the Green function for problem (9), so any solution of the integral equation

$$
x(t)=\int_{t_{0}}^{\infty} G(t, s) r(x(s), s) d s
$$

satisfies the boundary value problem (9). Now, we can state

Theorem 8. Assume that $A \in L(X)$ is such that $\operatorname{Sp}(A) \cap\{\alpha \in \mathbb{R}$ : $\alpha \leq 0\}=\emptyset$ and $r(x, t)=c(t) f(x)+b(t)$ where $c:\left[t_{0}, \infty\right) \rightarrow \mathbb{R}$ is continuous, $\lim _{|t| \rightarrow \infty}\|c(t)\|=0, b:\left[t_{0}, \infty\right) \rightarrow X$ is continuous and bounded, and $f:$ $X \rightarrow X$ is completely continuous. Then BVP (9) has at least one solution provided

$$
\limsup _{\|x\| \rightarrow \infty} \frac{\|f(x)\|}{\|x\|}<\frac{v w}{\|c\|_{\infty}\left(N_{1} w+N_{2} v\right)} .
$$

Pr o of. From inequality (10) we see that assumptions (ii)-(iv) of Theorem 3 are satisfied. As in the previous theorem assumptions (v), (vi) are satisfied, so the operator $S$ defined by the right-hand side of (11) is completely continuous.

Now, we show that it satisfies condition (2) of Theorem 4. By inequality (10),

$$
\begin{aligned}
L & =\sup _{t \in\left[t_{0}, \infty\right)} \int_{t_{0}}^{\infty}\|G(t, s)\| d s \leq \sup _{t \in\left[t_{0}, \infty\right)} \int_{t_{0}}^{\infty}\left(N_{1} e^{-v\left(t+s-2 t_{0}\right)}+N_{2} e^{-w|t-s|}\right) d s \\
& =\sup _{t \in\left[t_{0}, \infty\right)}\left(-\left.\frac{N_{1}}{v} e^{-v\left(t+s-2 t_{0}\right)}\right|_{s=t_{0}} ^{\infty}+\left.\frac{N_{2}}{w} e^{-w(t-s)}\right|_{s=t_{0}} ^{t}-\left.\frac{N_{2}}{w} e^{-w(s-t)}\right|_{s=t} ^{\infty}\right) \\
& =\sup _{t \in\left[t_{0}, \infty\right)}\left(\frac{N_{1}}{v} e^{-v\left(t-t_{0}\right)}+\frac{N_{2}}{w}-\frac{N_{2}}{w} e^{-w\left(t-t_{0}\right)}+\frac{N_{2}}{w}\right)=\frac{N_{1}}{v}+\frac{N_{2}}{w},
\end{aligned}
$$


whence, by (12),

$$
\limsup _{\|x\| \rightarrow \infty} \sup _{t \in T} \frac{\|c(t) f(x)+b(t)\|}{\|x\|} \leq\|c\|_{\infty} \limsup _{\|x\| \rightarrow \infty} \frac{\|f(x)\|}{\|x\|}<\frac{v w}{N_{1} w+N_{2} v}<\frac{1}{L} .
$$

Then from Theorem 4 we obtain the assertion.

\section{References}

[1] J. Banaś, Measures of noncompactness in the space of continuous tempered functions, Demonstratio Math. 14 (1981), 127-133.

[2] Yu. L. Daletskiŭ and M. G. Kreı̆n, Stability of Solutions of Differential Equations in a Banach Space, Nauka, Moscow, 1970 (in Russian).

[3] J. Diestel and J. J. Uhl, Jr., Vector Measures, Amer. Math. Soc., Providence, R.I., 1977.

[4] A. Hammerstein, Nichtlineare Integralgleichungen nebst Anwendungen, Acta Math. 54 (1929), 117-176.

[5] B. Przeradzki, The existence of bounded solutions for differential equations in Hilbert spaces, Ann. Polon. Math. 56 (1992), 103-121.

[6] K. Yosida, Functional Analysis, Springer, Berlin, 1974.

Faculty of Mathematics

University of Łódź

Banacha 22

90-238 Łódź, Poland

E-mail: stanczr@imul.uni.lodz.pl

Reçu par la Rédaction le 3.4.1997

Révisé le 17.11.1997 et le 25.3.1998 